\title{
APRENDIZAJE Y VIDA. CONSTRUCCIÓN, DIDÁCTICA, EVALUACIÓN Y CERTIFICACIÓN DE COMPETENCIAS EN EDUCACIÓN DESDE EL ENFOQUE SOCIOFORMATIVO
}

\author{
Lewis Herney García Mora \\ Especialista en intervención con familias \\ Magister en educación y desarrollo humano \\ Profesor $\mathrm{y}$ asesor pedagógico vinculado al \\ Centro de Estudios en Educación \\ Universidad Santo Tomas, Bucaramanga
}

\begin{tabular}{ll}
\hline AUTORES & Juan Antonio García Fraile; Nelly Milady López Rodríguez y Rosalba del Ángel Zúñiga \\
\hline EDITORIAL & Pearson \\
\hline PÁGINAS & 200 \\
\hline ÁREA EL CONOCIMIENTO & Educación \\
\hline EDICIÓN & Primera edición 2014 \\
\hline CIUDAD DE EDICIÓN & México \\
\hline
\end{tabular}

Formación por competencias desde el enfoque socioformativo: Aprendizajes para la vida, escrito por los ya coequiperos en este tema Juan Antonio García Fraile y Nelly Milady López Rodríguez quienes se acompañan en esta oportunidad de Rosalba del Ángel Zúñiga, es un texto que permitirá a docentes e interesados en el tema de formación por competencias profundizar en tópicos asociados al diseño y gestión de los procesos de formación para el desarrollo de competencias en educación desde el enfoque socioformativo. El cual presentan acompañando de gráficos, ejemplos una perspectiva práctica y con cierta rigurosidad, de tal forma, que el lector pueda acceder a la definiciones conceptuales fundamentales como a su aplicación práctica en contextos de educación básica, media y superior.

El libro muestra un recorrido que parte desde la presentación general de diferentes enfoques de abordaje de la noción de competencia y la manera en que ésta ha migrado hasta consolidarse como un concepto propio del contexto educativo. Desarrolla la comprensión y aplicación del enfoque socioformatvo, presentando, sin temor, las críticas más frecuentes que se la hacen al enfoque de competencias y finaliza con un capítulo dedicado al sistema de validación y certificación de competencias educativas y laborales en México. En su desarrollo los autores permiten al lector adentrarse en un escenario al parecer trajinado pero que viene a consolidarse como una visión integradora, pertinente y pragmática en el contexto de la educación formal.

El texto en general se concentra en la definición del enfoque socioformatvo por competencias sin dejar de reconocer la existencia el aporte de otras miradas que sirvieron, en el mejor de los casos, como referente para esta innovación educativa que pretende hacer emerger desde los propios contextos educativos una mirada propia, dinámica, flexible, pertinente y practica que trascendiera la visión instrumentalista de la planeación educativa tradicional. Para este efecto, los autores proponen principios rectores que contribuyen a la consolidación de este enfoque y que se orientan desde la clara idea de una educación centrada en el aprendizaje de los estudiantes como un proceso de construcción individual y colectiva en contextos determinados que requieren de ambientes colaborativos y de una relación particular entre el docente y el estudiante; una visión Neohumanista que 
reivindica los elementos de idoneidad y del proyecto ético de vida que las competencias implican y que brindan al proceso educativo la posibilidad de permitir al sujeto que aprende un crecimiento personal que parte de la potencialidades de cada individuo y de cada grupo en contexto; la noción de transdisciplinariedad, que provee una mirada integrativa y relacional del conocimiento que trasciende miradas reduccionistas tal como lo propondría Edgar Morín, para afincarse en concepciones complejas, que contribuyen a una comprensión más amplia de la realidad y de sus fenómenos para abordarlos y resolver las problemáticas que en ella se encuentran, privilegiando de esta manera el desarrollo de estrategias de enseñanza como el aprendizaje basado en proyectos $(A B P), y$ los Proyectos formativos integradores (PFI); la ya mencionada teoría dela complejidad que deviene de la propuesta Moriniana, que propende por trascender la mirada asignaturista, lineal y tradicional por una visión sistémico integradora complexa, orientada también dese una perspectiva Bertalanffyana que permite comprender el contexto global desde múltiples versiones.

Con estos referentes, los autores se permiten distinguir, describir y denominar los diferentes elementos humanos y materiales del proceso de Enseñanza, aprendizaje como un sistema de gestión de calidad del aprendizaje.

En un primer Capítulo: Origen y configuración de las competencias, los autores plantean que en la actualidad el enfoque de competencias es asumido por muchos países y que a partir de ahí se han desarrollado grandes aportes para su formación y evaluación. No obstante, reconocen que son pocos los estudios orientados a esclarecer su construcción conceptual y al establecimiento de acuerdos para definir un marco teórico de referencia común (Soto, 2002; Tobón, 2009).

Reconocen que sus aportes se basan en desarrollos metodológicos y conceptuales de

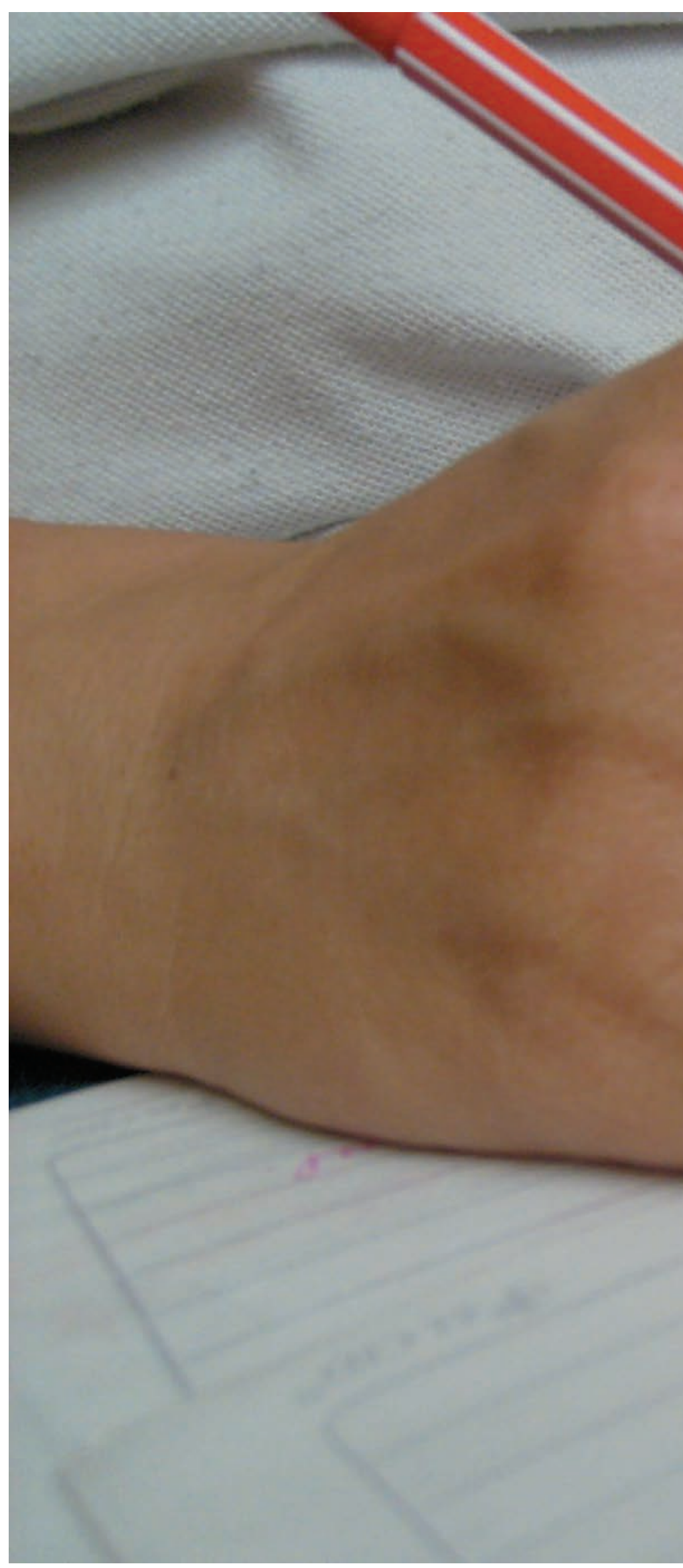


investigadores contemporáneos como Rial, 1997; García Fraile, 2000; García Fraile y Tobón, 2008; García Fraile y Tobón, 2009 y García Fraile, Tobón y López, 2009, quienes proponen planteamientos relevantes a la hora de abordar las competencias y la gestión del currículo.

Para desarrollar este capítulo los autores, plantean que la noción de competencias corresponde, en primera medida a una propuesta romana proveniente del latín Cumpetere que significa el "ir al encuentro una cosa de otra, ser adecuado, pertenecer" (Argudin, 2005), para referirse a la administración de cargos y a la división que se generó de los mismos, tal como parece ser usado en la actualidad en algunos contextos organizacionales y educativos. Asimismo, recuerdan que también existe una concepción griega del termino competencia que se adquirió desde el nacimiento de la cultura occidental y que se relaciona con el "rivalizar" que dio origen a la mirada de "competir, competencia, competidos y competitividad" entre personas, grupos u organizaciones (García Fraile y Tobón 2008 y Tobón, 2009). Concepciones que hoy son más naturales a contextos organizacionales y deportivos. Esto para recordarnos que el concepto no pertenece a una moda o a un capricho, sino a una forma de entender ciertas formas de concepción cultural acaecidas desde tempo atrás.

Con estos antecedentes, los autores muestran que las acepciones del término competencia son utilizadas cotidianamente para referirse a enfrentamientos deportivos, a funciones laborales en una organización, a las funciones y facultades de una persona o institución, entre otros. Recuerdan que las competencias surgen tiempo atrás en el Derecho castellano del siglo XVI por influencia del derecho romano y las influencias del renacimiento; así mismo, comienzan a abordarse en disciplinas como la Lingüística Chomskiana, posteriormente aplicadas por la sociología, la filosofía y retomadas por la pedagogía, la psicología y la formación para el trabajo a partir de marcos y concepciones disi- miles. Presentan un desarrollo interesante y general de los aportes de disciplinas como la lingüística, la psicología conductual, la psicología cultural y cognitiva, la sociología, la formación para el trabajo y la pedagogía. Para concluir que el enfoque de competencias se ha desarrollado en lo conceptual desde diferentes aportes y se instala en los procesos educativos como un resultado por el interés de atender asuntos relacionados con la calidad educativa, influenciado por aspectos históricos que implicaron aspectos económicos, sociales, tecnológicos y políticos que redundaron en la búsqueda del análisis, sistematización, comprensión y aplicación idónea de conocimientos en problemas específicos. Ajustándose a esta comprensión histórica, y retomando los aportes de Morín (1999), respecto a los saberes necesarios para la educación de donde resaltan las competencias del saber conocer, el saber hacer e integran el saber ser con el saber convivir, terminan proponiendo que cada una de los saberes se relaciona respectivamente con las dimensiones Cognitiva, actitudinal y socioafectiva, propias del enfoque socioformativo de competencias.

En el segundo capítulo los autores se refieren a la implementación del enfoque socioformativo de las competencias en los procesos de enseñanza y aprendizaje, retomando definiciones de competencia desde la Psicolingüística, la psicología cognitiva y la psicología cultural que se centran en el saber hacer en contexto:

- Competencia entendida como un saber hacer o conocimiento implícito en un campo del actuar humano (Hernández, Rocha y Verano, 1991, p.14).

- Competencia entendida como acción situada. Que se define en relación con determinados instrumentos mediadores (Torrado, 1998,p.42).

- Competencia entendida como saber hacer en contexto, o conjunto de acciones que un estudiante realiza en un contexto par- 
ticular y que cumplen con las exigencias académicas del mismo (Pardo, 1998, p.18)

Refieren que la definición de Levy-Leboyer (2000, p.10) se encuentra mucho más centrada en la eficacia del comportamiento humano:

- Competencia entendida como repertorio de comportamientos que algunas personas dominan mejor que otras, lo que las hace eficaces en una situación determinada.

También retoman dos definiciones de competencia que se centran en describir sus características definiéndolas como conjunto y combinación de atributos:

- Competencia entendida como conjunto de actitudes, conocimientos y habilidades específicas que hacen a una persona capaz de llevar a cabo un trabajo o de resolver un problema particular (Oullet, 20017, p.37)

- Competencia entendida como combinación de atributos respecto del conocimiento y sus aplicaciones, aptitudes, destrezas y responsabilidades que describen el nivel o grado de suficiencia con que una persona es capaz de desempeñarlo (Gonzales y Wagenaar, 2003, p.80).

- Los autores afirman que a pesar que no son suficientemente abarcadores y claras pues no hacen del saber una acción integradora, y no dan cuenta de la estructura y dinámica entre sus componentes, pero sobre todo, no hacen alusión a la idoneidad, elemento que, para autores como Tobón, 2006; Tobón, 2009; García Fraile y Tobón, 2008, son esenciales, resaltando además que las definiciones descritas han dejado un mal legado de instrumentalización y fragmentación en la manera de comprender y abordar pragmáticamente la noción de competencias en el ámbito educativo, ejemplificando esto con uno de los proyectos más importantes en edu- cación superior como lo es el proyecto "Tuning" y sus similares dentro del marco de la convergencia del Espacio Europeo para la Educación (EEES).

Los autores proponen los siguientes aspectos como integrantes de un acuerdo conceptual frente a la definición de competencia:

- Articulación sistémico relacional de actitudes, conocimientos y procedimientos

- Desempeño en actividades y acciones concretas como en el análisis y resolución de problemas

- Referencia a la idoneidad en el actuar

- Integración de saberes

Teniendo en cuenta estos aspectos consideran que una definición más completa y que aporte innovación y transformación en los escenarios educativos orientados a la calidad es la que presenta Tobón y García Fraile (2008, p.47) quienes definen la competencia como: acciones integrales ante problemas del contexto con idoneidad y compromiso ético.

Definen la Actuación Integral como un plexus sistémico y complejo en el que se integran las dimensiones afectivas y motivacional, cognitiva y actitudinal articuladas por un compromiso ético. La Idoneidad hace alusión a tener en cuenta una visión crítica o tener en cuenta criterios de calidad para resolver un problema o realizar una acción compleja desde la efectividad y la pertinencia. El Compromiso Ético es definido como elemento central del enfoque propuesto y es el elemento que aporta un carácter innovador frente a la manera en que se abordan las competencias desde otras propuestas. Es el que en toda actuación, contexto y ante cualquier finalidad permite al sujeto analizar la pertinencia ética de acuerdo con sus valores y el imaginario social. Así, el elemento central se encuentra en la acción metacognitva que realiza el sujeto an- 
tes, durante y después de su desempeño para apropiarse de los aspectos positivos y negativos de su acción. En este sentido, la persona competente es aquella que pone como centro de su desempeño el compromiso ético consigo mismo, con los otros y con el contexto ecosistémico donde se encuentra y se asume.

Desde este enfoque propuesto, los procesos de formación centrados en el desarrollo de competencias desde el enfoque socioformatvo implican potenciar las capacidades de los seres humanos para aprender, analizar contextos y resolver problemas, implicando y aplicando una perspectiva investigativa a partir de un nivel progresivo de desarrollo de la idoneidad en relación con el desempeño. Así pues, el desarrollo de competencias se articula directamente con el desarrollo de un proyecto ético de vida, intención que se encuentra dentro del propósito general de la educación de seres humanos integrales. Todo esto puede, entonces, encontrarse inmerso en un proyecto educativo institucional en diferentes niveles de formación.

Tener en cuenta esta perspectiva desde una mirada ecosistémica permite a los directivos educativos, docentes y estudiantes tener más elementos para el desempeño del rol que a cada uno le convoca en cuanto al desarrollo humano y a la construcción de conocimiento aplicado para la resolución de problemas relevantes en contextos educativos, o productivos.

Respecto a la descripción de las competencias en el currículo y en las actividades de enseñanza aprendizaje, la propuesta de los autores incluye tres elementos fundamentales a la hora de integrar el modelo de competencias desde el enfoque socioformatvo en los procesos de gestión curricular: a) Formulación de las competencias con soporte en el análisis de problemas del contexto, b) Criterios de desempeño (Pautas para evaluar la competencia, construidos con base en el estudio del contexto y ar- ticulan el saber ser, el saber conocer y el saber hacer ), y c) Evidencias (Pruebas concretas y tangibles que dan cuenta del grado de idoneidad con el cual se posee la competencia). Así mismo, proponen elementos complementarios para complementar la descripción de las competencias: d) Dominios de competencia (Categorías generales para clasificar las competencias que tienen características similares facilitando su organización) y e) Ejes procesuales (Desempeños concretos que dan cuenta de la estructura de una competencia y posibilitan organizar los criterios, por ejemplo, por ámbitos, áreas o bloques).

De esta manera, proponen que las competencias se describen teniendo en cuenta: a) verbo de acción; b) objeto de conocimiento ${ }^{1}$; c) finalidad y d) condición de referencia o de calidad y presentan tablas descriptivas de los elementos referidos, así como diferentes ejemplos de aplicación de esta metodología en un contexto de salud mental. Así mismo, se presenta el proceso de establecimiento de una competencia en un proceso de diseño curricular en cuatro pasos:

1. Definición del perfil ocupacional: identificación de la ocupación de carreras.

2. Definición de los dominios de competencia: determinación de los dominios de competencia de una ocupación o profesión y se agrupan las competencias por áreas.

3. Estudio del contexto: análisis complejo y completo para determinar los problemas a abordar por parte del profesional egresado.

4. Dominios de competencia: descripción de las competencias en cada nivel o dominio. 
Refieren que existen tres tipos de com-petencias: las Competencias Básicas, las Competencias Genéricas y las Competencias Específicas.

- Competencias Básicas: esenciales para desenvolverse en diferentes contextos vitales de manera cotidiana.

- Competencias Genéricas: se abordan a lo largo del sistema educativo con diferentes grados de complejidad en función del curso, nivel o grado. Estas son comunes a una rama o disciplina de conocimiento.

- Competencias Específicas: diferentes de las genéricas pero propias de cada nivel educativo o profesión y le imprimen la identidad a cada uno de ellos.

Para ejemplificar estos elementos, presentan ejemplos en organizaciones graficas respecto de las competencias genéricas en el bachillerato y en educación superior en un programa de administración de empresas.

Respecto a las competencias del docente en su rol como facilitador del proceso del estudiante de aprender a aprender y como transformador que facilita la interpretación y la construcción del conocimiento por parte de los estudiantes, consideran la formación del docente como proceso fundamental y proponen como referente ocho competencias del perfil del docente de educación media en México de acuerdo al sistema de educación Pública SEP, dado que estas puedes ser aplicables a docentes en otros países y en diferentes niveles de formación: a) Organiza su formación continua permanente; b) Dominio y estructuración de saberes para facilitar experiencias de aprendizaje significativo; c) Planificación de los procesos de Enseñanza-Aprendizaje atendiendo el enfoque de competencias y su aplicación en diversos contextos; d) Practica de procesos de Enseñanza-Aprendizaje de manera efectiva, creativa e innovadora en su contexto institucional; e) Evalúa los procesos de Enseñanza-Aprendizaje con enfoque for- mativo; f) Construye ambientes para el aprendizaje autónomo y colaborativo; g) Contribuye a la generación de un ambiente que facilite el desarrollo sano e integral de los estudiantes $y$, h) Participa en los proyectos de mejora continua de su institución y apoya la gestión institucional.

Al referirse a la planificación didáctica del proceso de construcción e implementación de una competencia los autores describen una ruta formativa para la construcción, planeación y evaluación de competencias realizando una descripción de cada una de las fases del proceso acompañándose de gráficas y de formatos que facilitarían el trabajo y la comprensión para aquel docente o directivo que pretenda realizar este ejercicio que empieza con: a) Establecimiento del perfil profesional de egreso; b) los datos de identificación del programa; c) Definición del eje transversal; d) verificación del proceso de construcción de competencia; e) Definición de las competencias de una asignatura; f) Identificación de los problemas a resolver; g) Definición de las actividades con acompañamiento del docente y de trabajo independiente del estudiante; h) Definición de los criterios y evidencias de aprendizaje; i) Construcción de las competencias y la definición de los logros, aspectos a mejorar y el proceso de retroalimentación.

En el tercer capítulo los autores revisan las críticas frecuentes al enfoque de competencias y reconocen que aunque es valorado y reconocido por docentes, instituciones, investigadores e incluso por los sistemas educativos y laborales de algunos países, también presenta detractores y críticos. Entre los descritos se encuentran los argumentos de Pereda y Berrocal (1999, p.28) acerca de que las competencias no existen o que no se pueden estudiar científicamente; la creencia histórica que las competencias son un enfoque para lo laboral y no para la educación superior que debe priorizar en la investigación, lo cual desconfigura el sentido de la educación como tal. 
Los argumentos de investigadores y académicos acerca de las competencias son una moda, lo cual desconoce el trabajo de otros tantos que vienen desarrollando conceptual y metodológicamente distintas innovaciones rigurosas en este campo. Las criticas recientes al enfoque de competencias que lo describen como una construcción del capitalismo o neoliberalismo, sin tener en cuenta que este es un enfoque diverso y complejo que tiene diferentes perspectivas no solo la que se centra en los proceso laborales y de formación para el trabajo; así como las críticas más recientes de los enfoques constructivistas que se centran en descripciones poco argumentadas de autores e instituciones que adoptan a conveniencia elementos parciales del enfoque $o$ simplemente ajustan ligeramente los conceptos y denominaciones propios del enfoque.

Ya en el cuarto capítulo se refieren al proceso de certificación de competencias que se aplica en México a través del Sistema Nacional de competencias de las Personas impulsado por el Consejo Nacional de Normalización de Competencias Laborales denominado (CONOCER), instancia del Gobierno Federal, sectorizada por la secretaría de educación pública SEP, con un órgano gubernamental que incluye diferentes representantes del sector de los trabajadores, empresarios y del gobierno. En este apartado se describe el modelo de evaluación y certificación de competencias del conocer, el cual se basa en estándares de competencias con criterios de objetividad, transparencia, imparcialidad, excelencia y libre acceso. El proceso de certificación que realizan los centros asociados y certificados por el CONOCER se fundamenta en una evaluación diagnóstica, un proceso de formación o recapacitación, una evaluación concreta de desempeños, productos, actitudes, hábitos y valores, la emisión de un juicio de expertos que conlleva o no al proceso de certificación o de identificación de necesidades de fortaleza.

Lo anterior se centra mucho más en procesos propios del sector ocupacional pero provee ciertos beneficios para los empleadores y em-

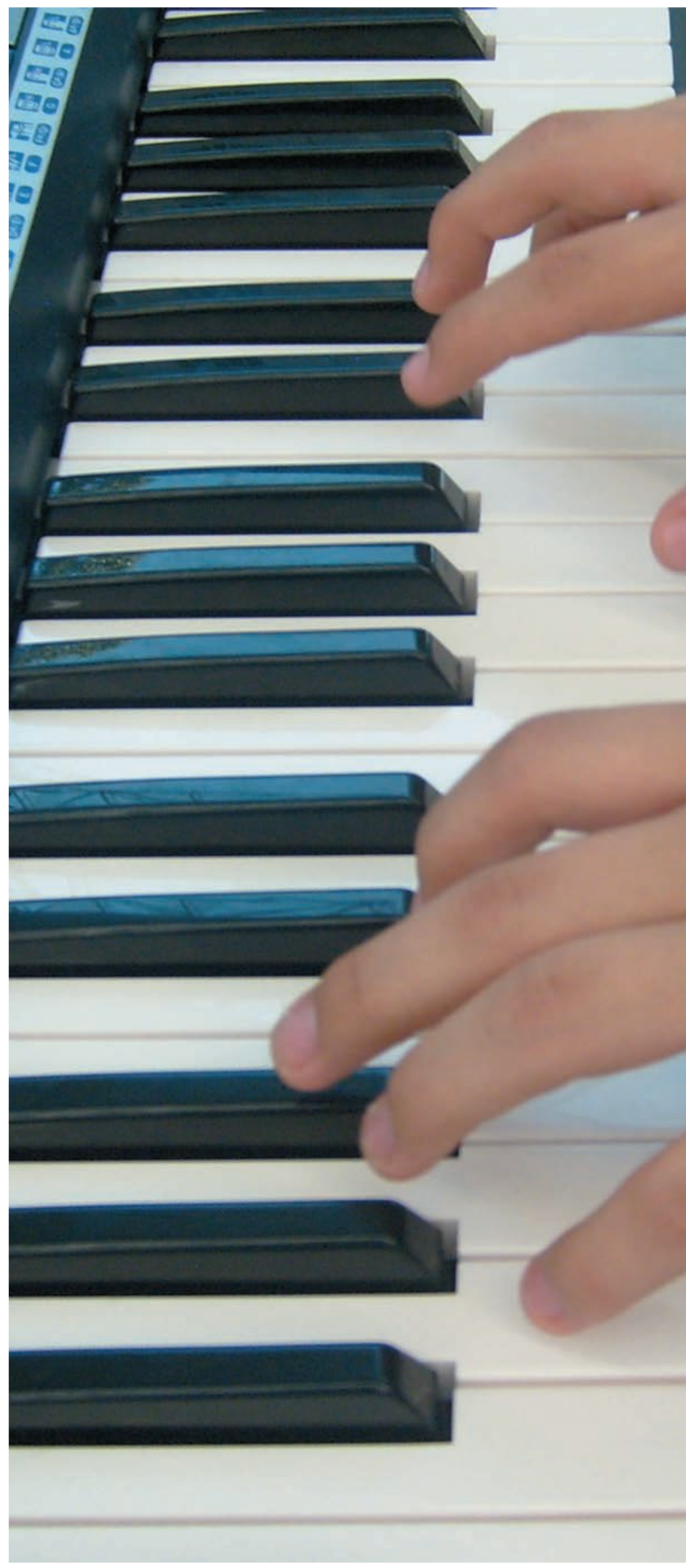



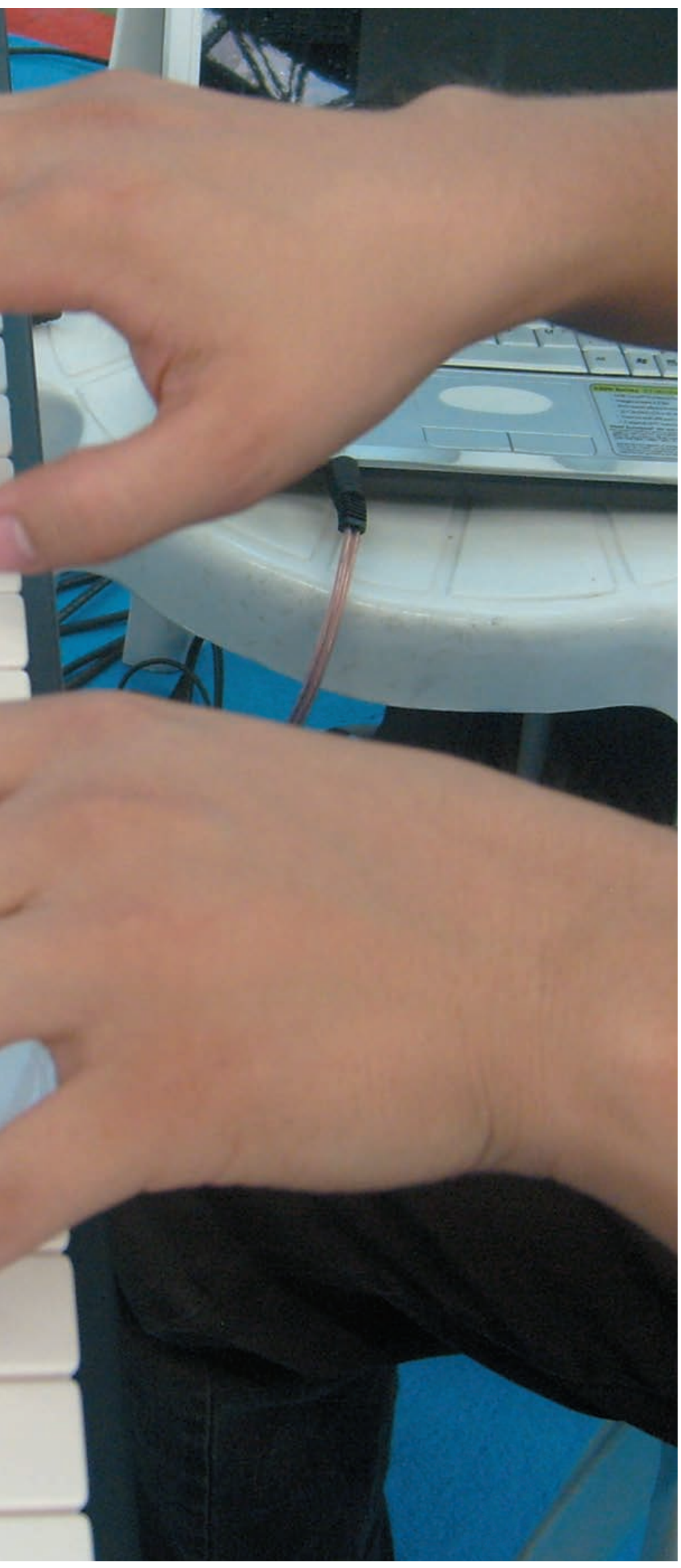

pleados entre los que se encuentran mayores oportunidades de ser vinculado y de cubrir vacantes en el sector productivo de México, contar con soportes para el empleado como para el empleador de tal manera que garanticen la experiencia e idoneidad del trabajo a realizar, la posibilidad de movilidad laboral a nivel nacional, estabilidad ocupacional, entre otras. En México, el sector educativo se encuentra permeado por estas lógicas de certificación y normalización y es así como las competencias de los alumnos se encuentran referidas en el Sistema Nacional de Bachillerato SNB y las de los docentes en la reforma educativa y sus leyes secundarias con el fin de alcanzar el desarrollo de las funciones educativas de docencia, investigación y la gestión escolar.

En esa medida el SNB que tiene por objetivo aumentar la calidad de la educación plantea definir tanto los perfiles del egresado como las acciones formativas tendientes a alcanzarlos adoptando el modelo de competencias Genéricas, Disciplinares básicas y extendidas y Profesionales básicas y extendidas, las cuales, permiten al estudiante alcanzar los niveles de estándares de competencias para desempeñar una función productiva o laboral. Para describir este proceso se presenta un ejemplo de estándar de competencia establecido por el comité de gestión para la formación para el trabajo en México.

Para finalizar, el texto presenta como anexos varios ejemplos del proceso de gestión curricular, didáctica y evaluación de competencias desde el enfoque socioformatvo aplicadas y existentes en contextos reales en el nivel de educación primaria, bachillerato, universitario para el área de ingenierías en sistemas, electrónica y administración en el contexto mexicano. Así mismo, presentan, a manera de ejemplo y demostración, las competencias docentes para los procesos de ejecución de cursos con en el enfoque de competencias y la aplicación de la metodología básica de investigación en el ámbito educativo aplicables en el contexto mexicano y validados por el conocer. 
Como colofón presentan un capítulo dedicado a las definiciones de términos propios al modelo de competencias desde el enfoque socioformatvo que fortalece la comprensión de los mismos para cualquier lector.

A manera de conclusión es importante resaltar que el texto se centra en el Hándicap o desempeño como aspecto integrador de las competencias y esta visión reúne elementos psicológicos, sociológicos y pedagógicos que se integran en el enfoque para consolidar una perspectiva interesante, pertinente y ante todo práctica.

Por su parte, a Integración del saber ser con el saber convivir se hace interesante pero parece desconocer la particularidad de dichos saberes, tal como lo propuso Morín en sus argumentos. Si bien es cierto van a aparecer integrados en el desempeño idóneo de un sujeto, la comprensión y abordaje integral merecen una comprensión de las relaciones entre cada elemento del complexus comportamental. Desde mi punto de vista, la definición de competencias debe estar orientada a la integración del saber hacer, el saber conocer, el saber comunicar y el saber convivir teniendo en cuanta que cada dimensión aporta elementos particulares e importantes. No obstante, es cierto que el modelo así lo propone y así viene trabajando, para lo cual es innegable su importante aporte e innovación.

El texto hace alusión al proceso de normalización y certificación de competencias en
México a través del sistema CONOCER, valdría la pena abordar también, de manera somera para hacer una comparación, los modelos de certificación de competencias en Colombia que realiza el Servicio Nacional de Aprendizaje SENA, así como el sistema europeo que opera en Francia a partir de la Comisión Nacional de Certificación Profesional, en el Reino Unido por la Autoridad de Cualificaciones y currículo, entre otras. Aunque reconozco que ese sería otro trabajo.

Posterior a la revisión, propongo que si bien el enfoque aplica a la formación de competencias en contextos institucionales, también se puede aplicar rigurosamente en otros escenarios contextuales, por ejemplo en la educación popular, la interacción social comunitaria, entre otros.

No obstante estas reflexiones finales, el texto presenta un esfuerzo importante por posicionar los procesos de formación por competencias desde el enfoque socioformatvo con cierto rigor epistémico, metodológico y practico, que le imprimen un nivel académico que lo hacen merecedor de ser tenido en cuenta como referente valido para abordar los procesos formativos en contextos institucionales. En este sentido, hago una invitación a abordar el texto para aproximarse cada vez más al modelo de competencias desde el enfoque socioformatvo aplicable a diferentes contextos y niveles educativos. 